\title{
Silibinin improves nonalcoholic fatty liver by regulating the expression of miR-122: An in vitro and in vivo study
}

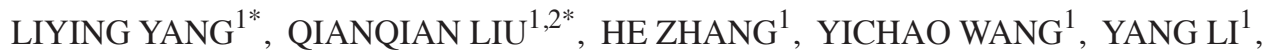 \\ SHUCHUN CHEN ${ }^{1}$, GUANGYAO SONG ${ }^{1}$ and LUPING REN ${ }^{1}$ \\ ${ }^{1}$ Endocrinology Department, Hebei General Hospital, Shijiazhuang, Hebei 050000; ${ }^{2}$ Hebei Research Institute for Endocrine and \\ Metabolic Diseases, Hebei North University, Zhangjiakou, Hebei 075000, P.R. China
}

Received June 18, 2020; Accepted January 12, 2021

DOI: $10.3892 / \mathrm{mmr} .2021 .11974$

\begin{abstract}
Silibinin is a flavonoid that improves fatty liver and insulin resistance. To elucidate the effect of silibinin on lipid deposition and the potential molecular mechanism, the present study conducted in vivo and in vitro experiments. In the in vivo experiments, mice were randomly divided into control, high-fat and silibinin groups, while HepG2 cells were randomly divided into control, palmitic acid intervention and silibinin intervention groups. The mRNA, protein and miR-122 expression associated with hepatic lipid metabolism were detected in each group. The results demonstrated that silibinin reduced the triglyceride content, miR-122 expression and the mRNA and protein expressions of fatty acid synthase (FAS) and acetyl-CoA carboxylase (ACC). Silibinin increased the mRNA and protein expression of carnitine palmitoyl transferase 1A (CPT1A). In the present study, HepG2 cells cultured with palmitate were treated with silibinin following overexpression of micro RNA (miR) 122. The results demonstrated that the mRNA and protein expression of FAS and ACC was increased, while that of CPT1A was decreased. Therefore, it could be deduced that silibinin improved lipid metabolism by reducing the expression of miR-122 and inhibiting the expression of miR-122 may be a new therapeutic target to improve fatty liver disease.
\end{abstract}

\section{Introduction}

Nonalcoholic fatty liver disease (NAFLD) is a metabolic stress-induced liver injury closely associated with insulin resistance (IR) and genetic susceptibility (1). The disease spectrum

Correspondence to: Dr Luping Ren, Endocrinology Department, Hebei General Hospital, 348 Heping West Road, Shijiazhuang, Hebei 050000, P.R. China

E-mail: renluping@hotmail.com

${ }^{*}$ Contributed equally

Key words: fatty liver, silibinin, miR-122, lipid metabolism, nonalcoholic fatty liver disease includes nonalcoholic simple fatty liver (NAFL), nonalcoholic steatohepatitis (NASH) and related liver cirrhosis and hepatocellular carcinoma (1). With economic development, unhealthy lifestyles and high-calorie diets are increasing, leading to an increase in the incidence of NAFLD. NAFLD affects $\sim 25 \%$ of the adult population worldwide and brings a huge burden to human wellbeing (2). Therefore, the prevention and treatment of NAFLD are under intensive focus worldwide.

The development of systemic biology and epigenetics has provided an in-depth insight into the association between genotype and phenotype of NAFLD (3). The epigenetic changes of NAFLD include histone modification, DNA methylation and changes in microRNAs (miRs/miRNAs). miRNAs are small, 18-25 nucleotide, non-coding, highly conserved regulatory RNAs that regulate gene expression at the post-transcriptional level $(3,4)$. These miRNAs regulate $>30 \%$ of human mRNAs and are also involved in a wide array of biological processes, including cell apoptosis, differentiation, development, proliferation and metabolism (5). The miRNAs that are closely associated with NAFLD have been extensively studied (3-5). miRNA-122 (miR-122) is the most abundant specific miRNA in the liver, accounting for about $70 \%$ of the total miRNA in adult liver (6). Previous studies have found that the expression of miR-122 in the serum of NAFLD patients is significantly increased $(4,5)$ and in vitro studies demonstrate that the overexpression of miR-122 enhances the activity of alanine aminotransferase (7).

The current treatment model for NAFLD is weight loss and comorbid management, without any specific drugs (8). In addition, improving the lifestyle is an effective treatment for NAFLD, but it can be difficult for individuals to abide by it. Some proposed medications have not shown significant efficacy and long-term safety (9). Silibinin is a flavonoid compound and the main component of silymarin in lipophilic milk thistle extract that is widely used for treating liver diseases (9). A previous study demonstrated that silibinin can enhance lipolysis by increasing the expression of triglyceride lipase, thereby reducing liver fat production, and can also downregulate the expression of genes, such as forkhead box O1, phosphoenolpyruvate carboxykinase and glucose-6-phosphatase to inhibit gluconeogenesis. Thus, silibinin improves high-fat-induced fatty liver and IR. The underlying mechanism may be to reduce visceral obesity, 
enhance lipolysis and inhibit gluconeogenesis (10). However, a previous study demonstrated that serum miR-122 levels and expression in NAFLD patients and fatty liver cells are increased, respectively (4), suggesting that silibinin and miR-122 are associated with lipid metabolism and NAFLD; however, the specific associations and mechanisms of action remain to be elucidated. Therefore, the present study constructed an NAFLD model using C57BL/6JC mice fed a high-fat diet (HFD) and the association between miR-122 and NAFLD and lipid metabolism in the liver was observed. Subsequently, silibinin was administered to this animal model to investigate the intervention effect of silibinin on the fatty liver of mice fed HFD and whether silibinin affected the expression of miR-122. Finally, in the NAFLD model palmitic acid (PA)-induced HepG2 cell line, miR-122 was regulated by transfection of mimics and the intervention was effectuated by silibinin to detect the expression levels of related lipid metabolism genes and proteins. Whether silibinin could improve liver lipid metabolism through the regulation of miR-122 was investigated. Therefore, the pathogenesis of NAFLD and putative therapeutic targets were revealed, providing a theoretical basis and novel ideas for clinical medication.

\section{Materials and methods}

Animal experiments. A total of 36 male C57BL/6JC mice (age, 7 weeks) were purchased from Beijing Vitong Lihua Experimental Animal Technology Co., Ltd., and reared in the barrier system for animal experiments in Clinical Research Center of Hebei Provincial People's Hospital. Mice were maintained at $20-25^{\circ} \mathrm{C}$ with $40-60 \%$ humidity and 12 -h light/dark cycles. The mice were randomly divided into two groups after 1 week of adaptive feeding: 12 mice in the control group (normal diet, ND) and 24 mice in the high-fat diet (HFD) group. In the HFD group, $60 \%$ calories were from fat, $20 \%$ from protein and $20 \%$ from carbohydrates. After 4 weeks, the HFD group was randomly divided into the HFD group and the HFD + silibinin (SIL) group with 12 mice each. The HFD + SIL group was administered $54 \mathrm{mg} / \mathrm{kg}$ body weight of SIL daily intragastrically and the ND and the HFD groups were administered with the same volume of normal saline intragastrically. The adult (human) dose was converted to the mouse dose in terms of body surface area based on the pharmacological test methodology (11). At a dose per body weight, the equivalent dose in mice was 9.1 times that in humans. The adult dose of silibinin is based on the manufacturer's instructions. Silibinin was mixed with food and fed to the mice (no anesthetic was employed).

At the end of the 8-week feeding, the mice were fasted overnight and anesthetized intraperitoneally with $1 \%$ sodium pentobarbital at $60 \mathrm{mg} / \mathrm{kg}$. The blood sample was withdrawn from the eyeball and serum collected by centrifugation at $865 \mathrm{x} \mathrm{g}$ for $20 \mathrm{~min}$ at $4^{\circ} \mathrm{C}$ and stored at $-80^{\circ} \mathrm{C}$ for later use. Acute massive blood loss in the orbital artery of mice was used and the mice succumbed immediately.

After the mice succumbed, the liver was removed and weighed. Several pieces of liver tissue were collected and frozen in liquid nitrogen before storage at $-80^{\circ} \mathrm{C}$ for subsequent reverse transcription-quantitative (RT-q) PCR and western blot analysis. Some liver tissues were placed in $10 \%$ neutral formaldehyde solution $(\mathrm{pH}=7.4)$ and fixed for hematoxylin and eosin (H\&E) staining. In addition, a portion of the liver tissue was embedded in optimal cutting temperature compound and stored at $-80^{\circ} \mathrm{C}$. Frozen sections were prepared for oil red $\mathrm{O}$ staining.

During the experiment, body weight, blood glucose and food intake of the mice were measured. The volume of blood collection for the glucose measurements was $2 \mu \mathrm{l}$.

Cell culture and treatment. Short tandem repeats were used to identify the HepG2 cells (Shanghai Saibaikang Bio Co., Ltd.) used in the present study. HepG2 cells are a hepatoma cell line. HepG2 cells were cultured in minimum essential medium (MEM, HyClone; Cytiva) containing $10 \%$ fetal bovine serum (FBS, Zhejiang Tianhang Biotechnology Co., Ltd.), at $37^{\circ} \mathrm{C}$ with $5 \% \mathrm{CO}_{2}$. A HepG 2 cell steatosis model was established by culture medium containing $0.25 \mathrm{mmol} / 1 \mathrm{PA}$ for $48 \mathrm{~h}$. HepG2 cells were transfected with miR-122-mimic (Guangzhou RiboBio Co., Ltd.) and negative control (Guangzhou RiboBio Co., Ltd.) and silibinin was used for cell interference. HepG2 cells were cultured in MEM medium of 5\% FBS for $48 \mathrm{~h}$ as miR-122 mimic transfection control. The transfection steps are described below. First, $1-5 \times 10^{5}$ cells were inoculated into a six-well plate containing complete medium with PA interference for $24 \mathrm{~h}$ to achieve the confluence of $50-60 \%$ for transfection. Second, $5 \mu 120 \mu \mathrm{M}$ miR-122 mimic with $120 \mu \mathrm{l}$ 1X Ribofect ${ }^{\mathrm{TM}}$ CP Buffer (Guangzhou RiboBio Co., Ltd.) was mixed with $12 \mu 1$ Ribo FECT ${ }^{\mathrm{TM}}$ CP Reagent and incubated at room temperature for $5 \mathrm{~min}$. Finally, the above transfection compounds were added to the $1863 \mu 1$ Lopti-MEM medium (Guangzhou RiboBio Co., Ltd.) and mixed gently. After transfection for 4-6 h, the medium containing 5\% FBS was changed and silybin was added for intervention for $24 \mathrm{~h}$ following transfection for 18-20 h (after $24 \mathrm{~h}$ of transfection) to collect the cells (namely, after transfection for $48 \mathrm{~h}$, all indicators were assessed for cell detection). The quantification of lipids by oil red $\mathrm{O}$ staining and triglyceride (TG) assay was performed to measure the TG level in HepG2 cells using a TG quantitative kit (Applygen Technologies, Inc.), according to the manufacturer's instructions.

Intraperitoneal glucose tolerance test (iPGTT). For iPGTT, mice were fasted for $12 \mathrm{~h}$ and then injected intraperitoneally with glucose $(2 \mathrm{~g} / \mathrm{kg})$, as described previously. Blood glucose from the mouse tail vein was measured at each of the following time points: $0,15,30,60$ and $120 \mathrm{~min}$. The tail tip of the mouse was cut short and blood was dripped onto a Roche rapid glucometer strip (Roche Diagnostics) to measure blood glucose.

Serum insulin determination. The insulin level of mice was determined by double anti-sandwich enzyme-linked immunosorbent assay (ELISA). A volume of $5 \mu \mathrm{l}$ of the standard and the sample was coated to the bottom of the plate, followed by addition of $75 \mu \mathrm{l}$ enzyme-labeled antibody. The reaction was incubated at room temperature for $120 \mathrm{~min}$. Finally, after washing, $100 \mu \mathrm{l}$ of the color substrate was added to each well and incubated in a room temperature shaker for $30 \mathrm{~min}$. The reaction was terminated with $100 \mu$ l termination solution and 
the absorbance was measured at the wavelength of $450 \mathrm{~nm}$ using an enzyme-labeled instrument (Thermo Fisher Scientific, Inc.). The standard curve was plotted according to the standard substance of different concentrations and the insulin level of the sample was calculated based on the standard curve and the absorbance of the sample.

Liver histology. H\&E staining was conducted to analyze the pathologic alterations. Briefly, liver tissues were fixed with $4 \%$ paraformaldehyde for $24 \mathrm{~h}$ at room temperature and then embedded in paraffin. Subsequently, tissues were sliced into $4 \mu \mathrm{m}$ sections followed by deparaffinization. Deparaffinization was performed by incubation with xylene for $40 \mathrm{~min}$, anhydrous ethanol for $10 \mathrm{~min}$ and $75 \%$ alcohol for $5 \mathrm{~min}$. Following washing with running water, tissues were stained with hematoxylin for 2-3 $\mathrm{min}$ and eosin for $1 \mathrm{~min}$ at room temperature. Stained tissues were observed using an OLFV-34CM/XE light microscope (Olympus Corporation).

The frozen fresh tissue was used for oil red $\mathrm{O}$ staining to analyze the accumulation of lipids in the liver. Liver tissues were frozen and cut into $8 \mu \mathrm{m}$ sections. The sections placed on slides and air-dried for $30 \mathrm{~min}$. Subsequently, tissues were fixed with paraformaldehyde for 15-20 min at room temperature. Following washing with running water, the sections were stained with oil red $\mathrm{O}$ for 8-10 min and counterstained with hematoxylin for 3-5 min at room temperature. Images were captured using an OLFV-34CM/XE light microscope. TG levels in liver tissues were determined according to the instructions of the TG quantitative kit (Applygen Technologies, Inc.).

RNA extraction and RT-qPCR measurement of $m R N A$ expression of fatty acid synthase (FAS), acetyl-CoA carboxylase $(A C C)$ and carnitine palmitoyl transferase $1 A$ (CPT1A). The mRNA expression of FAS, ACC and CPT1A in the liver and HepG2 cells were determined by RT-qPCR. First, the mRNA was extracted according to the instructions of the mRNA extraction and separation kit (Tiangen Biochemical Technology (Beijing) Co., Ltd.). Second, the reverse transcription reaction was performed on the Mastercycler gradient gene amplification instrument (Eppendorf) by referring to the instructions of the mRNA reverse transcription kit (Beijing Tiangen Biochemical Technology Co., Ltd.). Finally, the reaction system was set up according to the instructions of the mRNA amplification kit and amplification was performed on the ABI 7500 PCR instrument (Applied Biosystems; Thermo Fisher Scientific, Inc.). The following thermocycling conditions were used for qPCR: Pre-denaturation at $95^{\circ} \mathrm{C}$ for $15 \mathrm{~min}$; 40 cycles of denaturation at $95^{\circ} \mathrm{C}$ for $10 \mathrm{sec}$, annealing at $58^{\circ} \mathrm{C}$ for $20 \mathrm{sec}$ and extension at $72^{\circ} \mathrm{C}$ for $32 \mathrm{sec}$. cDNA synthesis and qPCR were performed according to the manufacturer's protocols. Subsequently, the data were analyzed using the ABI 7500 fluorescence quantitative PCR analysis software (Applied Biosystems; Thermo Fisher Scientific, Inc.). $\beta$ actin was set as the internal reference gene and the baseline was set as 3-15 cycles, following which the $\mathrm{Ct}$ values of each sample amplification were obtained. The control group samples were set as standard 1 and the relative quantitative value (RQ value) of the expression level of each target gene in each sample was obtained according to the equation, $R Q=2^{-\Delta \Delta C q}$ (12).
The following primer sequences were synthesized by Invitrogen (Thermo Fisher Scientific, Inc.): FAS forward, 5'-AGAACCTCCAGTCGTGTGAG-3' and reverse, 5'-GGG CATGGAGGGACTTGAAT-3'; ACC forward, 5'-ATGGGC GGAATGGTCTCTTTC-3' and reverse, 5'-TGGGGACCT TGTCTTCATCAT-3'; CPT1A forward, 5'-GACTCCGCT CGCTCATTCC-3' and reverse, 5'-TCGATGCCATCAGGG GTGAC-3'.

$R T$ - $q P C R$ to estimate the miR-122 expression. miR-122 expression in liver and HepG2 cells were determined by RT-qPCR. First, miRNA was extracted using the miRNA extraction and separation kit (Beijing Tiangen Biochemical Technology Co., Ltd.). Second, the reverse transcription reaction was performed on the Mastercycler gradient gene amplification instrument by referring to the instructions of the miRNA reverse transcription kit (Beijing Tiangen Biochemical Technology Co., Ltd.). Finally, the reaction system was set up according to the instructions of the miRNA amplification kit and performed on the ABI 7500 PCR instrument (Applied Biosystems; Thermo Fisher Scientific, Inc.). The primers for miR-122 were obtained from Guangzhou RiboBio Co. Ltd: miR-122 forward, 5'-AAA CGCCAUUAUCACACUAAAU-3' and reverse, 5'-UGGAGU GUGACAAUGGUGUUUG-3'. The following thermocycling conditions were used for qPCR: Pre-denaturation at $95^{\circ} \mathrm{C}$ for $15 \mathrm{~min}$; 40 cycles of denaturation at $94^{\circ} \mathrm{C}$ for $20 \mathrm{sec}$ and annealing/extension at $60^{\circ} \mathrm{C}$ for $34 \mathrm{sec}$. cDNA synthesis and qPCR were performed according to the manufacturer's protocols. Following amplification, the data were analyzed using ABI 7500 fluorescence quantitative PCR analysis software (Applied Biosystems; Thermo Fisher Scientific, Inc.). U6 (cat. no. CD201-0145; Tiangen Biotech Co., Ltd.) was set as the internal reference gene and the baseline was set as 3-15 cycles to obtain the $\mathrm{Ct}$ value of each sample and gene amplification. The control group samples were set as standard 1 and the RQ value of the expression level of each target gene in each sample was obtained according to the $\mathrm{RQ}=2^{-\Delta \Delta \mathrm{Cq}}(12)$, which was used for statistical analysis.

Western blot analyses of FAS, ACC and PT1A. The protein expression levels of FAS, ACC and CPT1A were determined by western blotting. Protein samples were extracted from liver tissue and HepG2 cells using RIPA buffer (Beijing Solarbio Science \& Technology Co., Ltd.) and protein concentrations were determined using the BCA protein assay kit (Applygen Technologies, Inc.). Then, an equivalent of $50 \mu \mathrm{g}$ protein was separated via 5\% SDS-PAGE (DYY-III; Beijing Liuyi Biotechnology Co., Ltd.) and transferred to the polyvinylidene fluoride membrane. Then, the membrane was blocked with 5\% Tris Buffered saline Tween (TBST; $5 \%$ Tween-20) in skimmed milk at room temperature for 2-4 h. Subsequently, the membrane was probed with primary antibodies (all 1:1,000) targeted against: FAS (cat. no. 3180S; Cell Signaling Technology, Inc.), ACC (cat. no. 3676S; Cell Signaling Technology, Inc.) rabbit monoclonal, CPT1A (cat. no. ARP44796_P050; Aviva Systems Biology), $\beta$-actin rabbit monoclonal (cat. no. AP0060; Bioworld Technology, Inc.) and GAPDH rabbit polyclonal (cat. no. AP0063; Bioworld Technology, Inc.) antibodies overnight at $4^{\circ} \mathrm{C}$ on a shaker (TS-1; Haimen Qilin Medical Instrument Factory), 
A

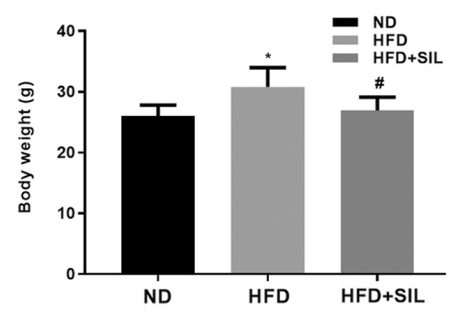

$\mathrm{C}$
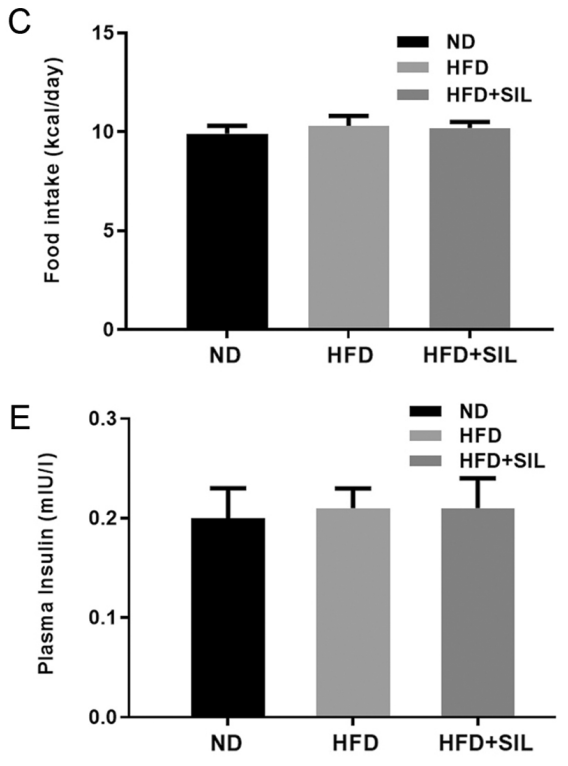

B

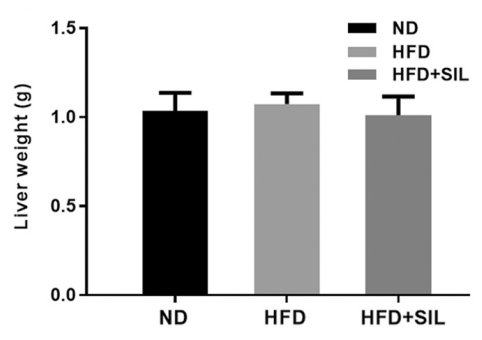

D

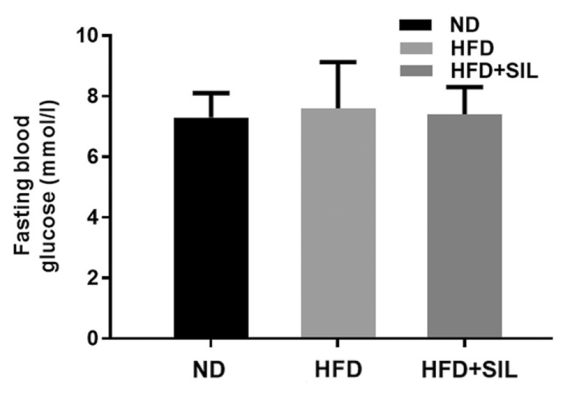

$\mathrm{F}$

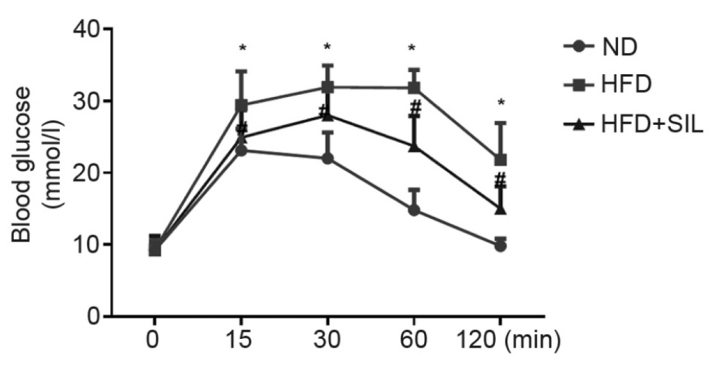

G

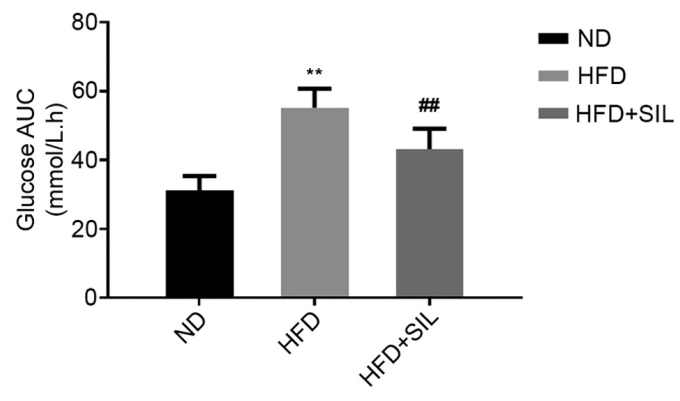

Figure 1. Comparison of body weight, liver wet weight, food intake, fasting glucose, fasting insulin, intraperitoneal glucose tolerance test and area under the glucose curve. (A) Body weight, (B) liver weight and (C) food intake of mice in each group. Data shown as mean \pm standard deviation; ${ }^{*}<0.05$ vs. ND and ${ }^{\#} \mathrm{P}<0.05$ vs. HFD. (D) Fasting Blood Glucose, (E) Plasma insulin, (F) intraperitoneal glucose tolerance test. (G) area under curve of glucose of mice in each group. Data shown as the mean \pm standard deviation. ${ }^{*} \mathrm{P}<0.05$ and ${ }^{* *} \mathrm{P}<0.01$ vs. ND, ${ }^{\#} \mathrm{P}<0.05$ and ${ }^{\# \#} \mathrm{P}<0.01$ vs. HFD. ND, normal diet; HFD, high-fat diet; SIL, silibinin.

followed by incubation with horseradish peroxidase-labeled goat anti-rabbit secondary antibody (cat. no. CW0103S; 1:5,000; Beijing Kangwei Century Biotechnology Co., Ltd.) at room temperature for $1 \mathrm{~h}$. Protein bands were visualized using enhanced chemiluminescence reagent (Thermo Fisher Scientific, Inc.) and an imaging system (Analytik Jena AG). GAPDH was used as the loading control. Protein expression was semi-quantified using ImageJ software (version 1.5.3; National Institutes of Health).

Statistical analyses. All data were analyzed by SPSS v21.0 software (IBM Corp.). Measurement data with normal distribution or close to normal distribution were expressed as mean \pm standard deviation, while measurement data without normal distribution were expressed as median (quartile). The t-test was used for the normal mean of two samples with homogeneous variance and a non-parametric rank-sum test was used for non-normal distribution or uneven variance. One-way analysis of variance was used for comparison among groups with post hoc tests Bonferroni for homogeneous variance and Tamhane's T2 for non-homogeneous variance. $\mathrm{P}<0.05$ was considered to indicate a statistically significant difference.

\section{Results}

Silibinin improves glucose tolerance in HFD mice. The bodyweight of mice in the HFD group was greater compared with mice in the silibinin and control groups $(\mathrm{P}<0.05$; Fig. 1A), although no significant differences were observed in liver weight and food intake between the three groups $(\mathrm{P}>0.05$; Fig. 1B and C). Also, no significant difference was observed in fasting glucose and fasting insulin among the three groups ( $\mathrm{P}>0.05$; Fig. 1D and E). The iPGTT was performed to verify the effect of silibinin on glucose metabolism-related 


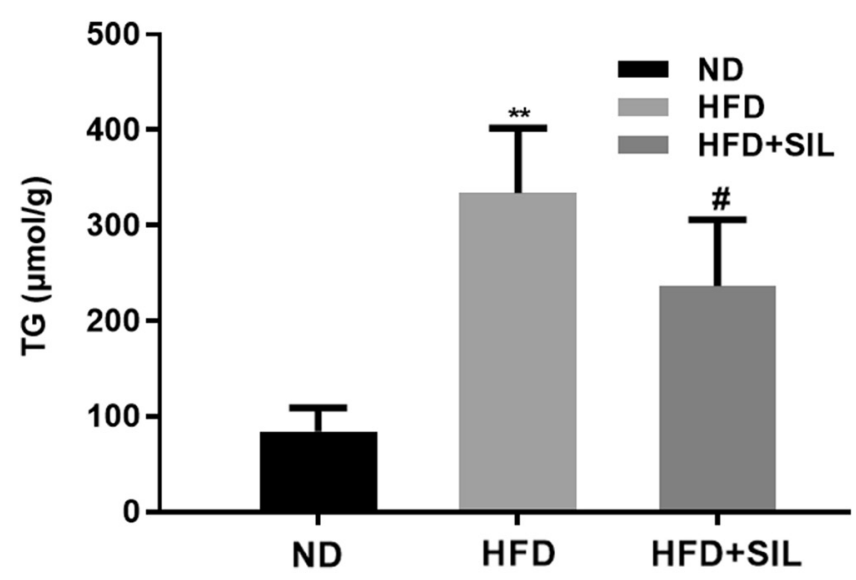

Figure 2. Effects of silibinin on TG content in mice liver. Data shown as the mean \pm standard deviation. ${ }^{* *} \mathrm{P}<0.01 \mathrm{vs}$. ND and ${ }^{\text {\#P}}<0.01$ vs. HFD. TG, triglyceride; ND, normal diet; HFD, high-fat diet; SIL, silibinin.

ND

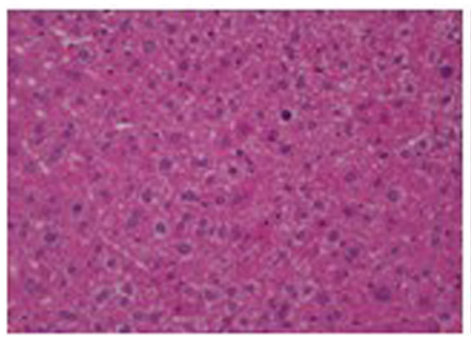

ND

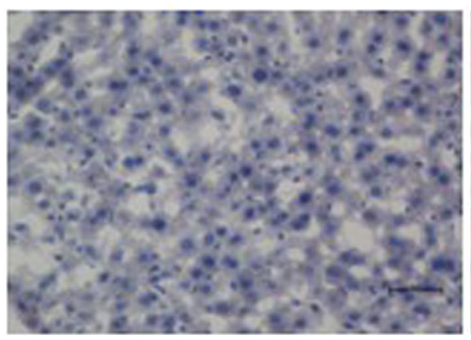

HFD

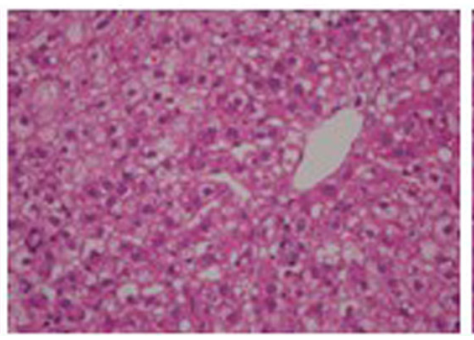

HFD

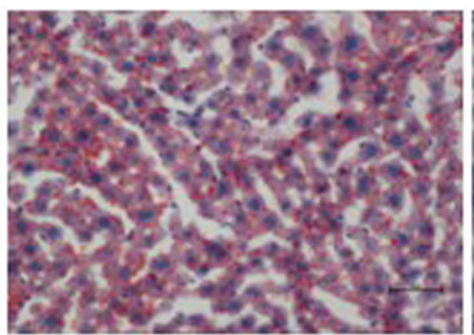

HFD+SIL

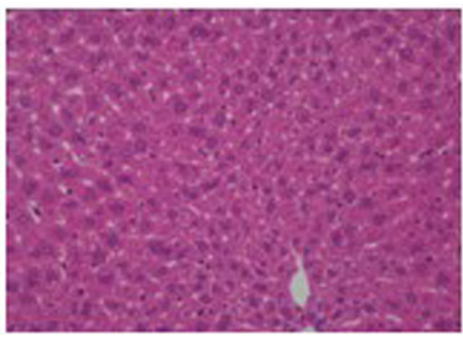

HFD+SIL

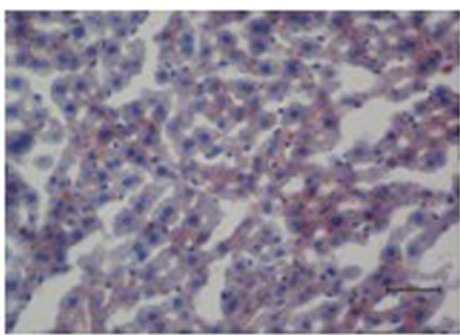

Figure 3. Histopathological examination of the mouse livers. Hematoxylin and eosin staining and oil red $\mathrm{O}$ staining of mice liver tissue (magnification, $\mathrm{x} 400$ ). ND, normal diet; HFD, high-fat diet; SIL, silibinin.

abnormalities. The blood glucose of the HFD group increased significantly at 15, 30, 60 and $120 \mathrm{~min}$ as compared with the ND group and the area under the blood glucose curve increased significantly $(\mathrm{P}<0.05$; Fig. $1 \mathrm{~F}$ and $\mathrm{G})$.

Silibinin treatment reduces hepatic steatosis induced by HFD. The liver TG content was significantly increased in the HFD group compared with the normal diet group $(\mathrm{P}<0.01)$, while that in the silibinin group was significantly reduced compared with the HFD group ( $\mathrm{P}<0.01$; Fig. 2). Light microscopy and histological examination of tissue sections stained with $\mathrm{H} \& \mathrm{E}$ and oil red $\mathrm{O}$ were performed to determine whether silibinin affected the hepatic lipid accumulation in mice. The results of H\&E staining demonstrated a severe ballooning degeneration of liver hepatocytes in the HFD group and a reduction of hepatic steatosis in the silibinin group. Oil red $\mathrm{O}$ staining demonstrated a large number of lipid droplets in the HFD group, whereas lipid droplets in the HFD+SIL group were markedly reduced compared with the HFD group. The oil red O staining confirmed the changes in the H\&E-stained sections (Fig. 3). The lipid accumulation in the liver was induced by fat, which was reduced by silibinin after feeding for 4 weeks. The gene expression of the key enzymes involved in hepatic lipid metabolism was quantified by RT-qPCR and western blotting. Compared with the normal diet group, the mRNA and protein expressions of FAS and ACC were increased in the HFD group $(\mathrm{P}<0.01)$, while that of $\mathrm{CPT} 1 \mathrm{~A}$ was decreased $(\mathrm{P}<0.01)$. Compared with the HFD group, the mRNA and protein expressions of FAS and ACC were decreased in the silibinin group and that of CPT1A was increased (P<0.01; Fig. 4).

Expression of miR-122 in mice livers of each group. Compared with the normal diet group, the expression of miR-122 was significantly increased in the HFD group $(\mathrm{P}<0.01)$. Compared with the HFD group, the expression of miR-122 was significantly decreased in the silibinin group $(\mathrm{P}<0.01$; Fig. 5). 
A

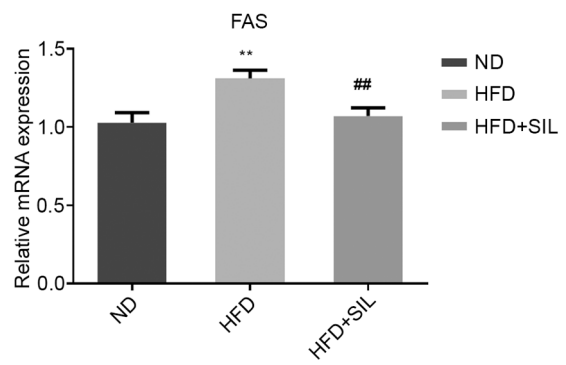

C

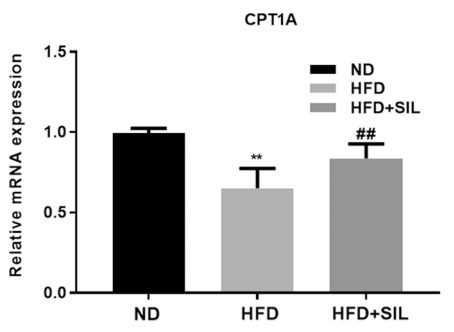

E

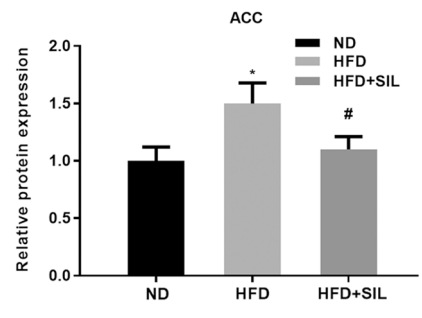

B

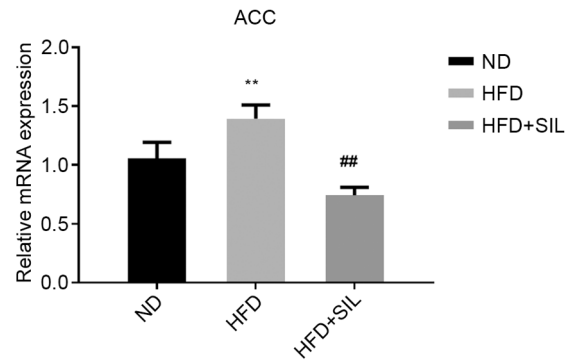

D

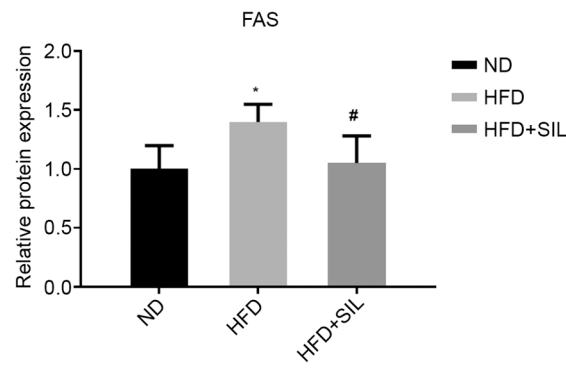

$\mathrm{F}$

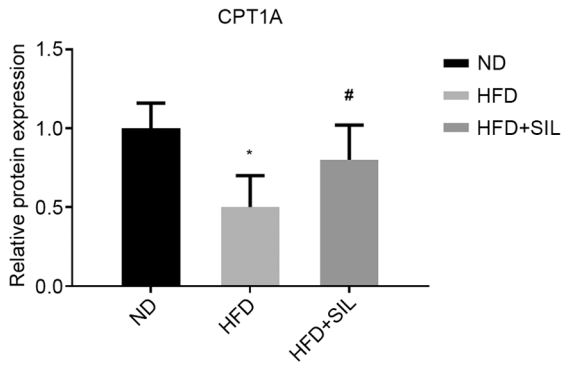

G

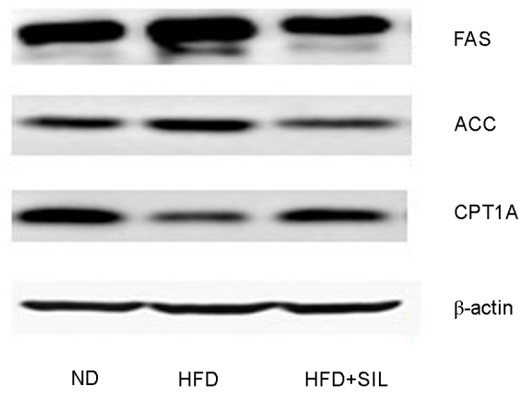

Figure 4. Effects of silibinin intervention on mRNA and protein expression levels of FAS, ACC and CPT1A in mice livers. Relative mRNA expression of (A) FAS, (B) ACC and (C) CPT1A in livers. Data shown as mean \pm standard deviation. Relative protein expression of (D) FAS, (E) ACC and (F) CPT1A in liver. (G) Western blot analysis of protein expression. Data shown as the mean \pm standard deviation. ${ }^{*} \mathrm{P}<0.05$ and ${ }^{* *} \mathrm{P}<0.01$ vs. ND, ${ }^{~} \mathrm{P}<0.05$ and ${ }^{\# \#} \mathrm{P}<0.01$ vs. HFD. FAS, fatty acid synthase; ACC, acetyl-CoA carboxylase; CPT1A, carnitine palmitoyl transferase 1A; ND, normal diet; HFD, high-fat diet; SIL, silibinin.

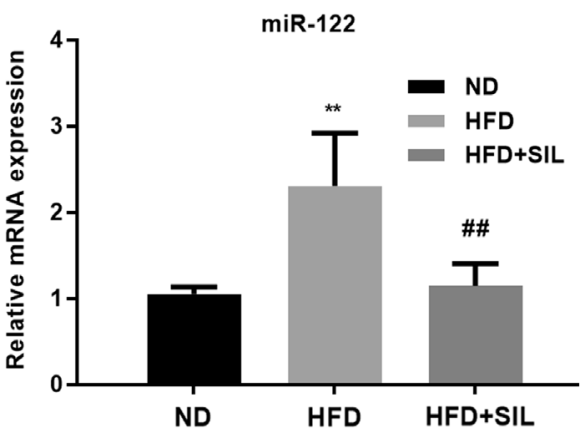

Figure 5. Expression of miR-122 in liver of each group. Relative hepatic expression of miR-122. Data shown as the mean \pm standard deviation. ${ }^{* *} \mathrm{P}<0.01 \mathrm{vs}$. ND and ${ }^{\# / P}<0.01$ vs. HFD. miR, microRNA; ND, normal diet; HFD, high-fat diet; SIL, silibinin.
Silibinin treatment improves hepatic lipid metabolism in HepG2 cells. Compared with the Con group, the TG content in HepG2 cells increased significantly after PA intervention $(\mathrm{P}<0.01)$. Compared with the PA group, TG content in the $\mathrm{PA}+$ SIL group was significantly decreased $(\mathrm{P}<0.01$; Fig. $6 \mathrm{~A})$. Oil red O staining is illustrated in Fig. 6B. Cells in the Con group had no obvious lipid droplets, while cells in the PA group had a large number of red staining lipid droplets and lipid droplets in the PA + SIL group were significantly reduced compared with the PA group.

Effects of Silibinin on miR-122 expression in HepG2 cells cultured in normal medium and in the presence of $P A$. Compared with the Con group, the miR-122 expression in the 
A

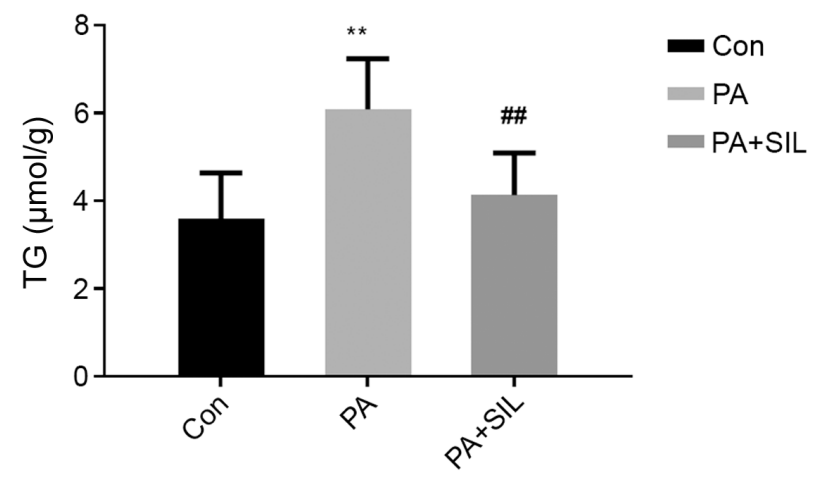

B

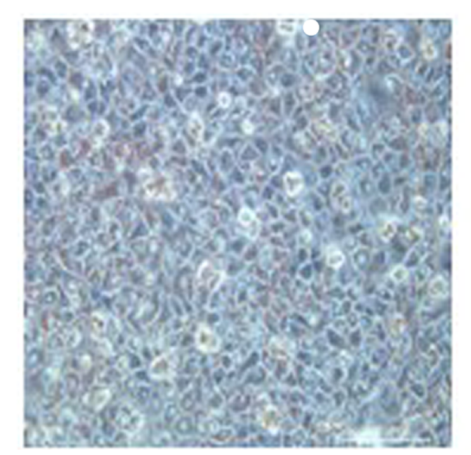

PA

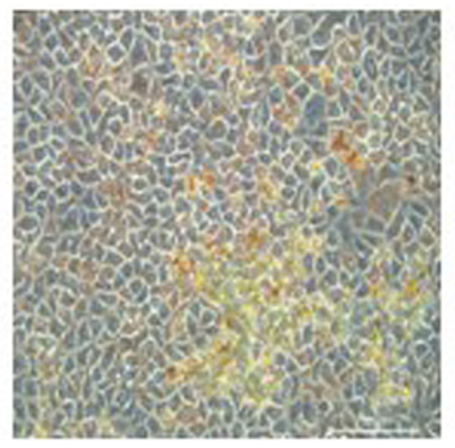

$\mathrm{PA}+\mathrm{SIL}$

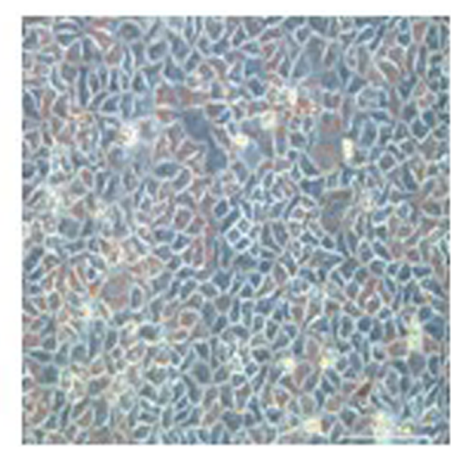

Figure 6. Effects of silybin on lipid deposition in HepG2 cells cultured with PA. Effect of silibinin intervention on (A) TG level and lipid accumulation in HepG2 cells exposed to PA. (B) Oil red O staining indicated that silibinin obviously reduced lipid accumulation in PA-induced HepG2 cells (magnification, $\mathrm{x} 200$ ). Data shown as mean \pm standard deviation. ${ }^{* *} \mathrm{P}<0.01$ vs. Con and ${ }^{\# \#} \mathrm{P}<0.01$ vs. PA. PA, palmitic acid; TG, triglyceride; Con, control; SIL, silibinin.
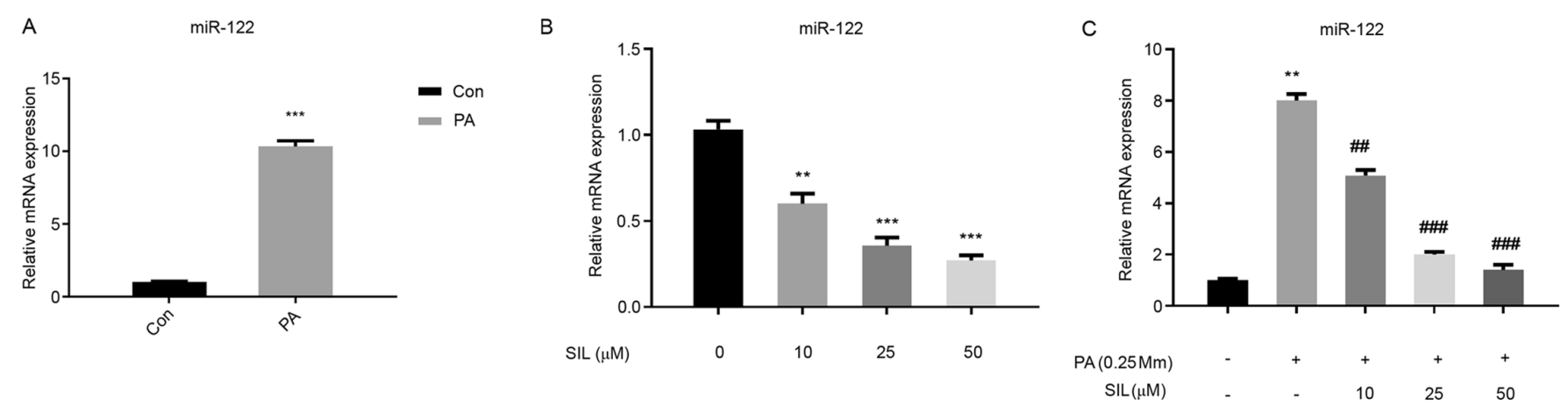

Figure 7. Effects of silibinin on miR-122 expression in HepG2 cells cultured in normal medium and HepG2 cells cultured with PA. (A) Expression of miR-122 in HepG2 cells treated with or without PA. (B) The effect of silibinin on transcriptional level of miR-122. (C) Effect of silibinin on transcriptional level of miR-122 in HepG2 cells treated with PA. Data shown as the mean \pm standard deviation. ${ }^{* *} \mathrm{P}<0.01$ vs. Con, ${ }^{* * * *} \mathrm{P}<0.001$ vs. Con, ${ }^{\# \# P} \mathrm{P}<0.01$ vs. $\mathrm{PA},{ }^{\# \# \#} \mathrm{P}<0.001$ vs. PA, Con, control; PA, palmitic acid; miR, microRNA.

PA intervention group was significantly increased $(\mathrm{P}<0.01$; Fig. 7A). Following SIL intervention in ordinary medium cells at different doses, the expression of miR-122 decreased significantly with the increase in SIL dose (Fig. 7B). Compared with the PA group, the expression of miR-122 in the PA + SIL group decreased $(\mathrm{P}<0.01)$ and the decreasing range was elevated with the increase in SIL dose (Fig. 7C).

Effects of silibinin intervention on mRNA and protein expression levels of FAS, ACC and CPT1A in HepG2 cells cultured with PA. Compared with the Con group, the mRNA and protein expression of FAS and ACC increased in the PA group $(\mathrm{P}<0.01)$, while that of CPT1A decreased significantly
$(\mathrm{P}<0.01)$. Compared with the PA group, the mRNA and protein expressions of FAS and ACC decreased in the PA + SIL group $(\mathrm{P}<0.01)$ and that of $\mathrm{CPT} 1 \mathrm{~A}$ was increased significantly $(\mathrm{P}<0.01$; Fig. 8).

Silibinin affects liver lipid metabolism by regulating miR-122 expression. Following miR-122-mimic transfection in HepG2 cells, the miR-122 mRNA level in the miR-122-mimic group was significantly higher than that in the control group (Empty; $\mathrm{P}<0.05$ ), indicating a successful miR-122-mimic transfection (Fig. 9A). Compared with the Con group, the mRNA expression of FAS and ACC increased in the PA group $(\mathrm{P}<0.01)$, 
A

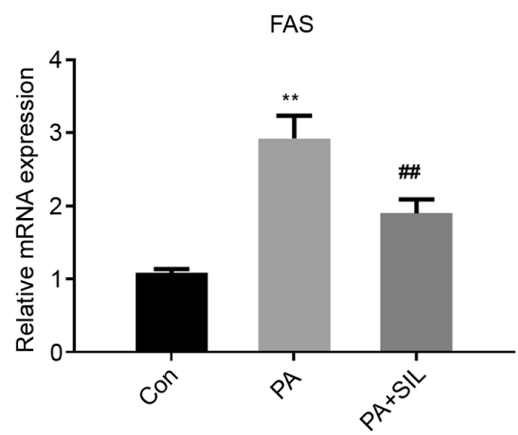

C

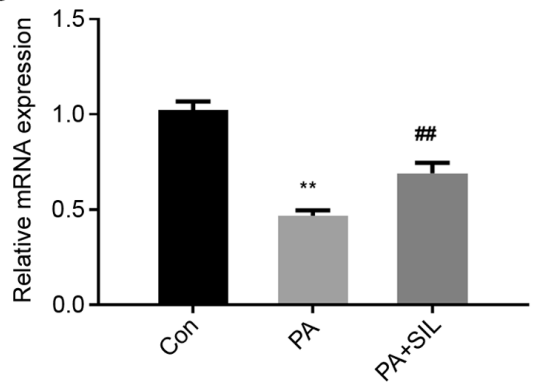

E

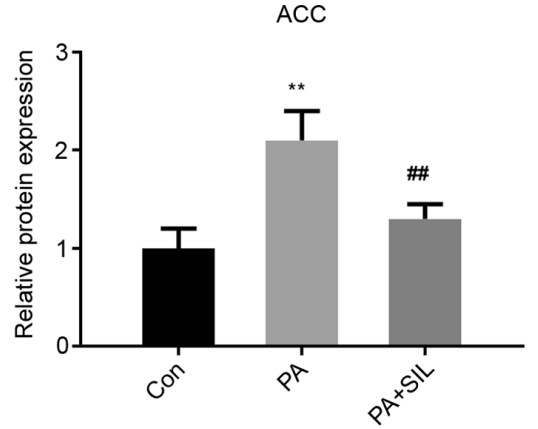

G

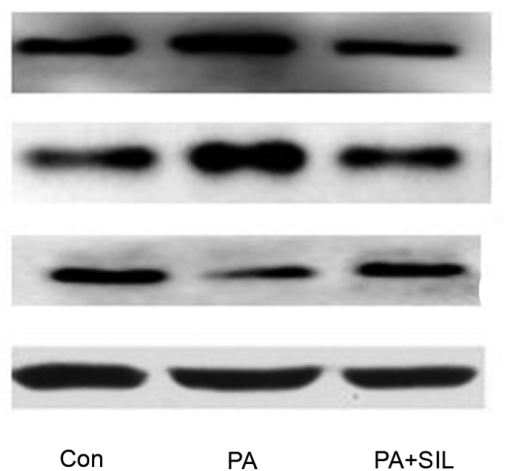

B

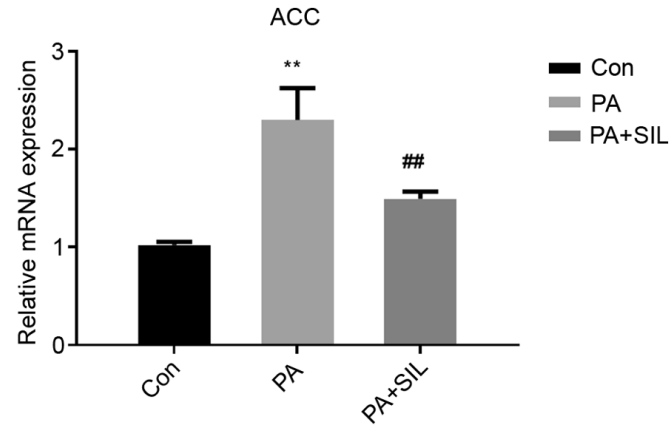

D

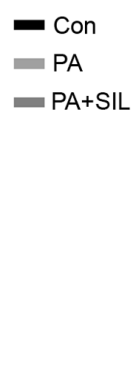

D

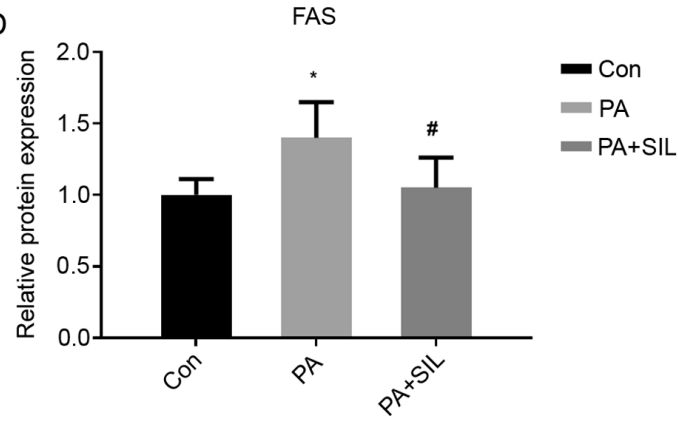

$\mathrm{F}$

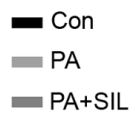

$F$

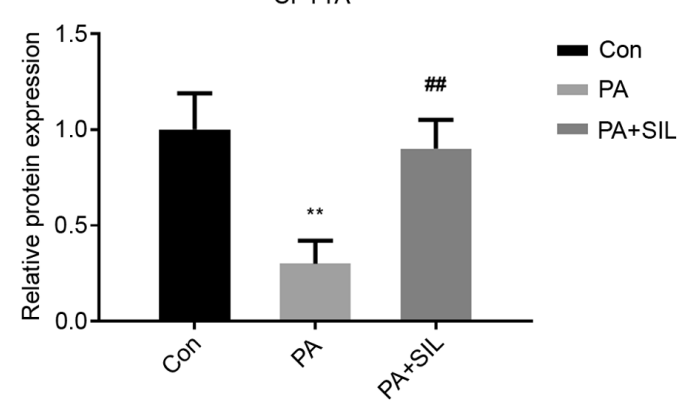

FAS

ACC

CPT1A

GAPDH

Figure 8. Effects of silibinin intervention on mRNA and protein expression levels of FAS, ACC and CPT1A in HepG2 cells cultured with PA. Relative mRNA expression of (A) FAS, (B) ACC and (C) CPT1A in HepG2 cells. Data shown as the mean \pm standard deviation. The relative protein expression of (D) FAS, (E) ACC and (F) CPT1A in HepG2 cells. (G) Western blot analysis of protein expression. Data shown as mean \pm standard deviation. ${ }^{*}<<0.05$ and ${ }^{* *} \mathrm{P}<0.01$ vs. Con, ${ }^{\#} \mathrm{P}<0.05$ and ${ }^{\# \#} \mathrm{P}<0.01$ vs. PA. miR, microRNA; FAS, fatty acid synthase; ACC, acetyl-CoA carboxylase; CPT1A, carnitine palmitoyl transferase $1 \mathrm{~A}$; PA, palmitic acid; Con, control; SIL, silibinin.

while that of CPT1A decreased $(\mathrm{P}<0.01)$. Compared with the PA group, the mRNA expression of FAS and ACC decreased in the PA + SIL group $(\mathrm{P}<0.01)$ and that of CPT1A increased $(\mathrm{P}<0.01)$. Following transfection with NCmiR-122mimic the mRNA expressions of FAS and ACC were decreased $(\mathrm{P}<0.01)$ and that of CPT1A increased $(\mathrm{P}<0.01)$ in the $\mathrm{PA}+\mathrm{SIL}+\mathrm{NCmiR}-122$ mimic group, compared with the PA group. Following transfection with miR-122 mimic, compared with the PA + SIL group and PA + SIL + NCmiR-122mimic group, the mRNA expression of FAS and ACC was significantly increased in the PA + SIL + miR-122 mimic group $(\mathrm{P}<0.01)$ and that of CPT1A was significantly decreased $(\mathrm{P}<0.01$; Fig. 9B-D). Compared with the Con group, the protein level of FAS and ACC in the PA group increased, while that of CPT1A decreased $(\mathrm{P}<0.01)$. Compared with the PA group, the protein expression of FAS and ACC in the PA + SIL group decreased 
A

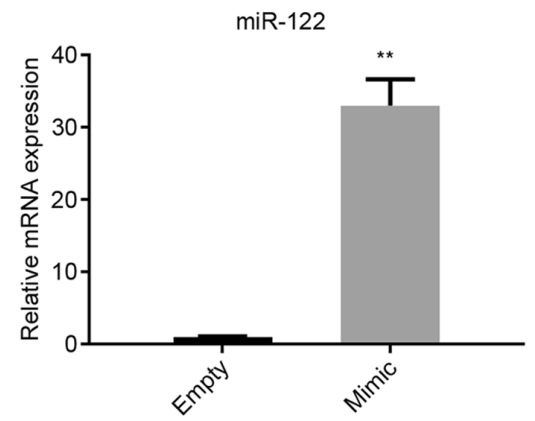

C

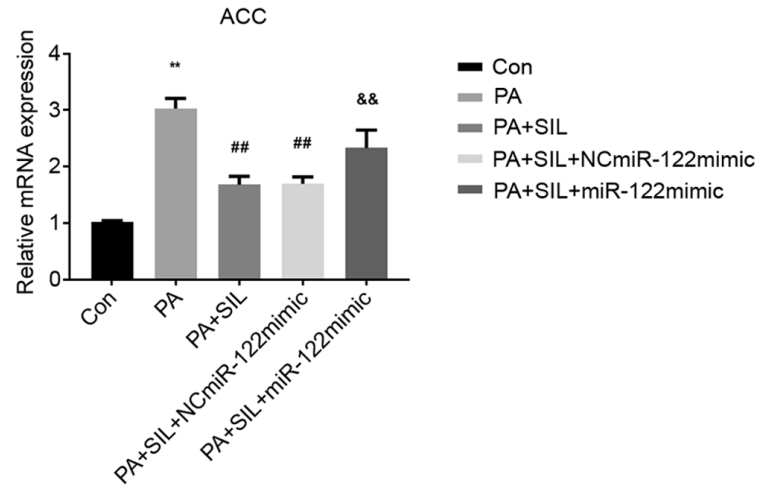

E

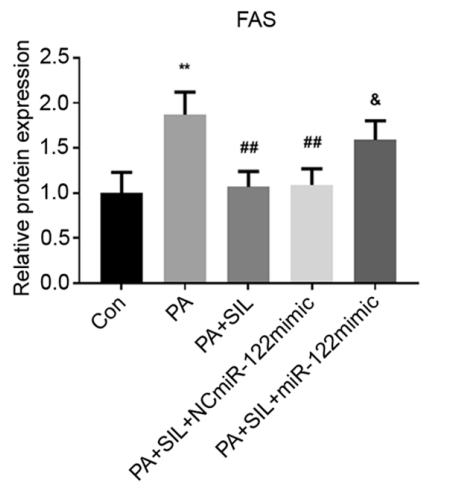

G

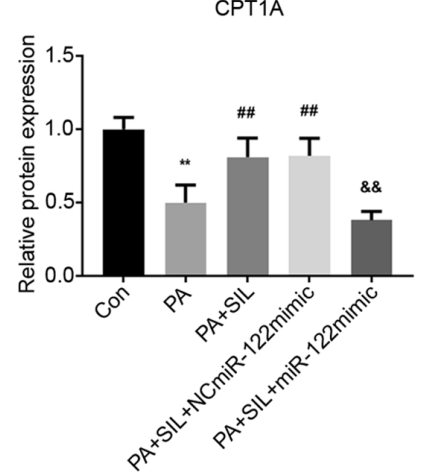

Empty

- Mimic

B

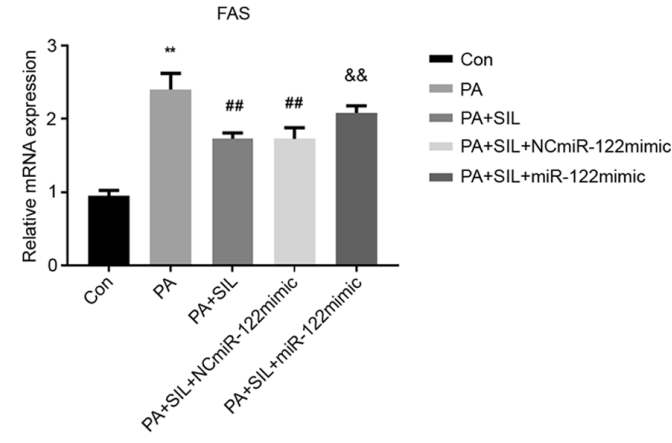

D

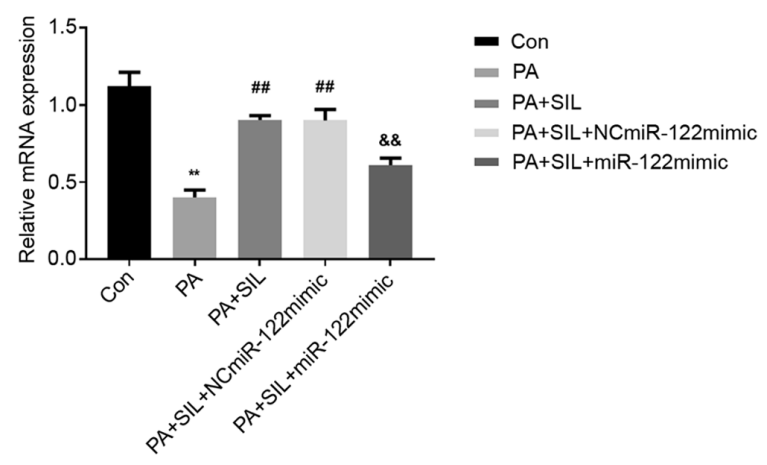

F
- Con

= PA

- PA+SIL+NCmiR-122mimic

- PA+SIL+miR-122mimic

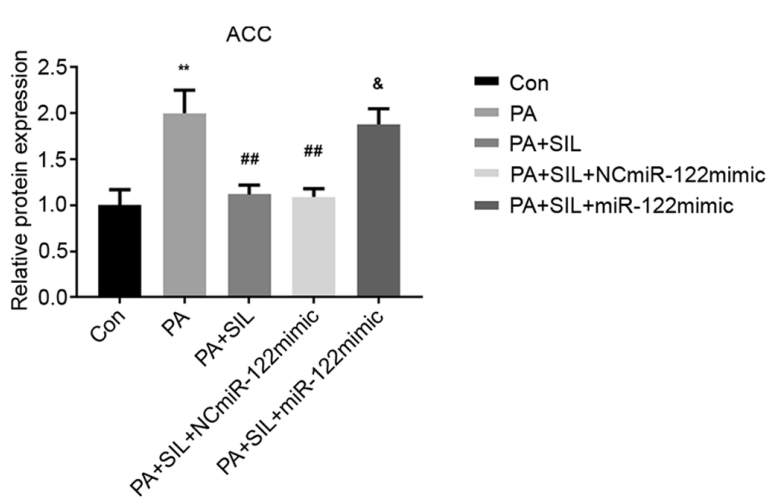

- PA

- PA+SIL

- PA+SIL+NCmiR-122mimic

- PA+SIL+miR-122mimic
H

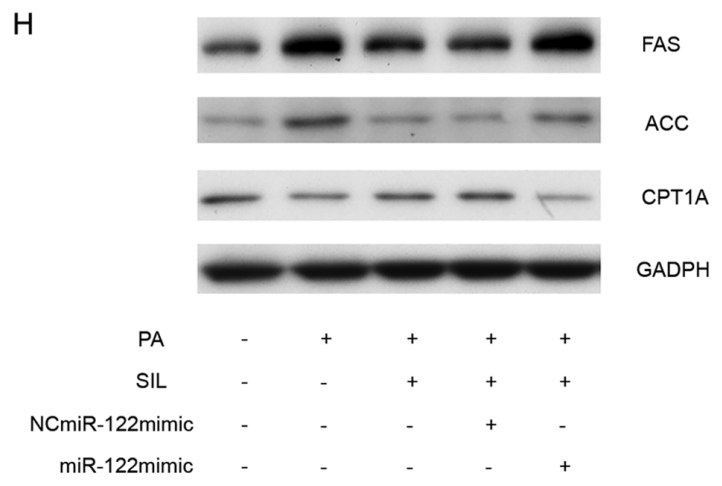

Figure 9. Silibinin affects liver lipid metabolism by regulating miR-122 expression. (A) Effect of miR-122 mimic on the level of miR-122 in HepG2. Data shown as the mean \pm standard deviation. ${ }^{* *} \mathrm{P}<0.01$ vs. Empty. The relative mRNA expression of (B) FAS, (C) ACC and (D) CPT1A in HepG2 cells following transfection. Data shown as the mean $\pm \mathrm{SD}$. ${ }^{* *} \mathrm{P}<0.01$ vs. con, ${ }^{\# \#} \mathrm{P}<0.01$ vs. $\mathrm{PA},{ }^{\& \&} \mathrm{P}<0.01$ vs. $\mathrm{PA}+\mathrm{SIL}$. The relative protein expression of (E) FAS, (F) ACC and (G) CPT1A in HepG2 cells following transfection. (H) Western blot analysis of protein expression. Data shown as the mean \pm standard deviation. ${ }^{* *} \mathrm{P}<0.01 \mathrm{vs}$. con, ${ }^{\# \#} \mathrm{P}<0.01$ vs. PA, ${ }^{\&} \mathrm{P}<0.05$ and ${ }^{\& \&} \mathrm{P}<0.01$ vs. PA + SIL and PA + SIL + NCmiR-122mimic. miR, microRNA; FAS, fatty acid synthase; ACC, acetyl-CoA carboxylase; CPT1A, carnitine palmitoyl transferase 1A; PA, palmitic acid; Con, control; NC, negative control; SIL, silibinin.

$(\mathrm{P}<0.01)$, while that of CPT1A increased $(\mathrm{P}<0.01)$. Following transfection with NCmiR-122mimic, the protein expressions of FAS and ACC were decreased $(\mathrm{P}<0.01)$ and that of CPT1A increased $(\mathrm{P}<0.01)$ in the $\mathrm{PA}+\mathrm{SIL}+\mathrm{NCmiR}-122 \mathrm{mimic}$ 
group compared with the PA group. Following transfection with miR-122 mimic, compared with the PA + SIL group and $\mathrm{PA}+\mathrm{SIL}+\mathrm{NCmiR}-122 \mathrm{mimic}$ group, the protein expression of FAS $(\mathrm{P}<0.05)$ and ACC $(\mathrm{P}<0.05)$ in the PA + SIL + miR-122 mimic group increased and that of CPT1A decreased significantly $(\mathrm{P}<0.01$; Fig. 9E-H).

\section{Discussion}

The increasing prevalence of NAFLD has become a public health challenge. It is a complex and multifactorial disease that involves numerous genetic, environmental and metabolic factors and is closely associated with obesity, type 2 diabetes and metabolic syndrome. The liver plays a key role in lipid metabolism. Fatty liver is the result of the accumulation of various lipids in liver cells (10). The following mechanisms may contribute to the development of the simple fatty liver disease: i) Increased dietary fat intake or increased visceral/subcutaneous adipose tissue lipolysis leads to the increase in free fatty acids; ii) Reduced free fatty acid oxidation; iii) Increase of de novo lipogenesis of the liver; and iv) Reduced low-density lipoprotein in the liver reduces the secretion of TGs (13). Some patients with NAFLD can develop steatohepatitis, liver fibrosis and cirrhosis (14). In addition, a previous study demonstrated that cardiovascular, malignant and liver-related morbidity and mortality risks are higher in patients with NAFLD (14). A number of studies have proved that silibinin increases insulin sensitivity, improves hepatic steatosis, reduces oxidative stress, protects the livers and improves metabolic syndrome (10,15-17). In the present study, silibinin was used to intervene in the HFD-induced NAFLD model in vivo and in vitro to explore the mechanism underlying silibinin intervention in NAFLD.

Changes in lifestyle and dietary structure increases the incidence of fatty liver, especially HFD and high-calorie diet (3). In vivo and in vitro studies have shown that high lipids lead to lipid deposition in fatty liver and liver cancer HepG2 cells of mice $(17,18)$. Simultaneously, the expression of lipid de novo synthesis indicators, FAS and ACC increase and the expression of fatty acid $\beta$ oxidation indicator CPT1A decreases $(18,19)$. In the present study, HFD-fed mice and $0.25 \mathrm{mmol} / 1 \mathrm{PA}$-treated HepG2 cells were successfully established NAFLD models in vivo and in vitro, respectively. It was found that high-fat intervention resulted in lipid deposition in mouse liver and HepG2 cells (expressed as H\&E-stained lipid droplet vacuoles and/or oil red $\mathrm{O}$ staining). In addition, the TG content in liver tissue and HepG2 cells and the expression of FAS and ACC increased, while the expression of CPT1A, an indicator of fatty acid oxidation, decreased. These findings suggested that high fat stimulates the de novo lipid synthesis in liver cells and inhibits mitochondrial oxidation of fatty acids at the animal and cellular levels. These results were similar to those of the aforementioned previous studies, which demonstrated that high-fat intervention causes lipid deposition, elevates the index of lipid synthesis and decreases the index of fatty acid oxidation.

HFD-fed mice and HepG2 cells with PA intervention represent a caloric overload state that leads to obesity, IR and liver steatosis. In the present study, silibinin reduced the lipid deposition in HepG2 cells and improved IR and hepatic lipid deposition in HFD mice. Animal experiments have demonstrated that silibinin improves the fatty liver of HFD mice by regulating liver glucose metabolism and improving IR (10). In vitro experiments have demonstrated that silibinin targets IRS-1/PI3K/Akt to improve IR and hepatic lipid deposition in an NAFLD cell model induced by PA. The present study conducted western blot analysis with a phosphor-AKT antibody using the same samples as described in Fig. 4D-G (20). Another study demonstrated that silibinin inhibits oxidative stress, reduces liver damage, regulates liver lipid homeostasis and protects the liver (17). The results of the present study were consistent with those of the previous studies that silibinin can improve lipid deposition and IR in an NAFLD model.

The present study also observed the mediating effect of miR-122 on hepatic lipid deposition induced by hyperlipidemia and the intervention effect of silibinin. miRNAs have been under intensive investigation in recent years and achievements have been made in the fields of cell growth, apoptosis, metabolism, inflammation, immune regulation and tumor (3-5). The role of miRNA in the development of fatty liver has also aroused interest. Feng et al (21) found that miR-200c expression is upregulated in HFD-fed rats and fatty acid-treated HepG2 cells. Calo et al (22) demonstrated that following miR-21 knockdown in the liver, glucose intolerance, lipid deposition and obesity are improved in HFD mice. In addition, for a non-invasive test, serum miR-34a, miR-122 and miR-192 have been identified as potential biomarkers for NAFLD (23). As the most abundant miRNA expressed in the liver, miR-122 has been widely studied. A number of studies have shown that miR-122 mediates the occurrence and development of the fatty liver. For example, Miyaaki et al (4) found that miR-122 knockdown resulted in the upregulation of lipid metabolism genes, including FAS, HMGCR reductase and sterol binding element-binding protein, and miR-122 expression is significantly upregulated in simple fatty liver degeneration or NASH and positively correlated with the severity of hepatocyte steatosis. Su et al (24) fed mice HFD for 10 weeks and found that the liver size increased 3.6 times in these mice along with the increased expression of FAS and decreased expression of CPT1A. Naderi et al (25) used the liver biopsy of clinical samples and found that the miR-122 expression in the NAFLD group was significantly upregulated compared with the normal obese control group. Miyaaki et al (4) demonstrated that the miR-122 level in the liver of patients with severe steatosis $(>33 \%)$ was significantly higher compared with patients with mild steatosis $(<33 \%)$. In the present study, the expression of miR-122 in HFD-fed mice and HepG2 cells with PA intervention increased, that of FAS and ACC also increased, while that of CPT1A decreased. Silibinin intervention reduced the expression of FAS and ACC and increased the expression of CPT1A, while the expression of miR-122 was downregulated. Thus, the findings of the present study were consistent with the above studies and both in vivo and in vitro experimental results indicated that the expression of miR-122 is positively associated with the occurrence and development of NAFLD.

In the present study, miR-122 expression of HepG2 cells in normal medium and PA medium was significantly decreased after intervention with silibinin at different concentrations in 
HepG2 cells and the reduction of miR-122 was dose-dependent with silibinin concentration. It was hypothesized that silibinin reduced the expression of miR-122. In order to further explore the effect of silibinin on miR-122 in lipid metabolism, the present study used a miR-122 mimic and transfection technique to overexpress miR-122, followed by silibinin interference in HepG2 cells cultured with PA. Consequently, the effect of silibinin on improving lipid deposition was inhibited and mRNA and protein expressions of FAS and ACC were increased, while that of CPT1A was decreased, suggesting that silibinin improves lipid metabolism by reducing miR-122 expression. At present, there are no relevant studies on the regulation of miR-122 expression by silibinin, to the best of the authors' knowledge, and only a few studies have confirmed that silybin changes the expression of miRNA in cancer cells $(26,27)$.

In summary, silibinin improved lipid metabolism by reducing miR-122 expression. miR-122 may become a new therapeutic target for improving fatty liver and the molecular mechanism requires further study.

\section{Acknowledgements}

Not applicable.

\section{Funding}

The present study was supported by the Natural Science Foundation of Hebei Province (grant no. H2019307114).

\section{Availability of data and materials}

The datasets used and/or analyzed during the current study are available from the corresponding author on reasonable request.

\section{Authors' contributions}

LY and QL conceived the study, participated in data collection, analysis and interpretation, and drafted the manuscript. $\mathrm{HZ}$ and YW contributed to collecting samples and materials, and analyzing data. YL, SC, GS and LR participated in the analysis and interpretation of the results. All authors read and approved the final manuscript and consented to publish this manuscript. LY and QL confirmed the authenticity of all the raw data.

\section{Ethics approval and consent to participate}

All experimental procedures were approved by the Animal Ethic Board of Hebei Research Institute for Endocrine and Metabolic Diseases (approval number 202024) and were in accord with China's National Code of the Animal Care for Scientific Experimentation. Hebei Research Institute for Endocrine and Metabolic Diseases is affiliated to Hebei General Hospital.

\section{Patient consent for publication}

Not applicable.

\section{Competing interests}

The authors declare that they have no competing interests.

\section{References}

1. Committee of Hepatology, Chinese Research Hospital Association; Fatty Liver Expert Committee, Chinese Medical Doctor Association; National Workshop on Fatty Liver and Alcoholic Liver Disease, Chinese Society of Hepatology; National Workshop on Liver and Metabolism, Chinese Society of Endocrinology, Chinese Medical Association: Expert recommendations on standardized diagnosis and treatment for fatty liver disease in China (2019 revised edition). Zhonghua Gan Zang Bing Za Zhi 27: 748-753, 2019 (In Chinese).

2. Yu Y, Cai J, She Z and Li H: Insights into the epidemiology, pathogenesis, and therapeutics of nonalcoholic fatty liver diseases. Adv Sci (Weinh) 6: 1801585, 2018.

3. Liu XL, Cao HX and Fan JG: MicroRNAs as biomarkers and regulators of nonalcoholic fatty liver disease. J Dig Dis 17: 708-715, 2016

4. Miyaaki H, Ichikawa T, Kamo Y, Taura N, Honda T, Shibata H, Milazzo M, Fornari F, Gramantieri L, Bolondi L and Nakao K: Significance of serum and hepatic microRNA-122 levels in patients with non-alcoholic fatty liver disease. Liver Int 34: e302-307, 2014.

5. Yamada H, Suzuki K, Ichino N, Ando Y, Sawada A, Osakabe K, Sugimoto K, Ohashi K, Teradaira R, Inoue T, et al: Associations between circulating microRNAs (miR-21, miR-34a, miR-122 and miR-451) and non-alcoholic fatty liver. Clin Chim Acta 424: 99-103, 2013.

6. Chai C, Rivkin M, Berkovits L, Simerzin A,Zorde-Khvalevsky E, Rosenberg N, Klein S, Yaish D, Durst R, Shpitzen S, et al: Metabolic circuit involving free fatty acids, microRNA 122 , and triglyceride synthesis in liver and muscle tissues. Gastroenterology 153: 1404-1415, 2017.

7. Yamada H, Ohashi K, Suzuki K, Munetsuna E, Ando Y, Yamazaki M, Ishikawa $\mathrm{H}$, Ichino $\mathrm{N}$, Teradaira $\mathrm{R}$ and Hashimoto S: Longitudinal study of circulating miR-122 in a rat model of non-alcoholic fatty liver disease. Clin Chim Acta 446: 267-271, 2015.

8. Singh S, Osna NA and Kharbanda KK: Treatment options for alcoholic and non-alcoholic fatty liver disease: A review. World J Gastroenterol 23: 6549-6570, 2017.

9. Salomone F, Godos J and Zelber-Sagi S: Natural antioxidants for non-alcoholic fatty liver disease: Molecular targets and clinical perspectives. Liver Int 36: 5-20, 2016.

10. Yao J, Zhi M, Gao X, Hu P, Li C and Yang X: Effect and the probable mechanisms of silibinin in regulating insulin resistance in the liver of rats with non-alcoholic fatty liver. Braz J Med Biol Res 46: 270-277, 2013.

11. Nair A, Morsy MA and Jacob S: Dose translation between laboratory animals and human in preclinical and clinical phases of drug development. Drug Dev Res 79: 373-382, 2018.

12. Livak KJ and Schmittgen TD: Analysis of relative gene expression data using real-time quantitative PCR and the 2(-Delta DeltaC(T)) method. Methods 25: 402-408, 2001.

13. Tilg $\mathrm{H}$ and Moschen AR: Evolution of inflammation in nonalcoholic fatty liver disease: The multiple parallel hits hypothesis. Hepatology 52: 1836-1846, 2010.

14. Lindenmeyer CC and McCullough AJ: The natural history of nonalcoholic fatty liver disease-an evolving view. Clin Liver Dis 22: 11-21, 2018.

15. Bouderba S, Sanchez-Martin C, Villanueva GR, Detaille D and Koceir EA: Beneficial effects of silibinin against the progression of metabolic syndrome, increased oxidative stress, and liver steatosis in Psammomys obesus, a relevant animal model of human obesity and diabetes. J Diabetes 6: 184-192, 2014.

16. Vecchione G, Grasselli E, Voci A, Baldini F, Grattagliano I, Wang DQ, Portincasa P and Vergani L: Silybin counteracts lipid excess and oxidative stress in cultured steatotic hepatic cells. World J Gastroenterol 22: 6016-6026, 2016.

17. Salamone F, Galvano F, Cappello F, Mangiameli A, Barbagallo I and Li Volti G: Silibinin modulates lipid homeostasis and inhibits nuclear factor kappa B activation in experimental nonalcoholic steatohepatitis. Transl Res 159: 477-486, 2012. 
18. Cui CX, Deng JN, Yan L, Liu YY, Fan JY, Mu HN, Sun HY, Wang YH and Han JY: Silibinin Capsules improves high fat diet-induced nonalcoholic fatty liver disease in hamsters through modifying hepatic de novo lipogenesis and fatty acid oxidation. J Ethnopharmacol 208: 24-35, 2017.

19. Salomone F, Barbagallo I, Godos J, Lembo V, Currenti W, Cinà D, Avola R, D'Orazio N, Morisco F, Galvano F and Li Volti G: Silibinin restores $\mathrm{NAD}^{+}$levels and induces the SIRT1/AMPK pathway in non-alcoholic fatty liver. Nutrients 9: 1086, 2017.

20. Zhang Y, Hai J, Cao M, Zhang Y, Pei S, Wang J and Zhang Q: Silibinin ameliorates steatosis and insulin resistance during non-alcoholic fatty liver disease development partly through targeting IRS-1/PI3K/Akt pathway. Int Immunopharmacol 17: 714-720, 2013.

21. Feng YY, Xu XQ, Ji CB, Shi CM, Guo XR and Fu JF: Aberrant hepatic microRNA expression in nonalcoholic fatty liver disease. Cell Physiol Biochem 34: 1983-1997, 2014.

22. Calo N, Ramadori P, Sobolewski C, Romero Y, Maeder C, Fournier M, Rantakari P, Zhang FP, Poutanen M, Dufour JF, et al: Stress-activated miR-21/miR-21* in hepatocytes promotes lipid and glucose metabolic disorders associated with high-fat diet consumption. Gut 65: 1871-1881, 2016.

23. Liu CH, Ampuero J, Gil-Gomez A, Montero-Vallejo R, Rojas A, Muñoz-Hernández R, Gallego-Durán R and Romero-Gómez M: miRNAs in patients with non-alcoholic fatty liver disease: A systematic review and meta-analysis. J Hepatol 69: 1335-1348, 2018.
24. Su D, Zhang R, Hou F, Chi J, Huang F, Yan S, Liu L, Deng Y, Wei Z and Zhang M: Lychee pulp phenolics ameliorate hepatic lipid accumulation by reducing miR-33 and miR-122 expression in mice fed a high-fat diet. Food Funct 8: 808-815, 2017.

25. NaderiM,Pazouki A,ArefianE,HashemiSM,Jamshidi-AdeganiF, Gholamalamdari O, Soudi S, Azadmanesh K, Samiee SM, Merat $\mathrm{S}$, et al: Two triacylglycerol pathway genes, CTDNEP1 and LPIN1, are down-regulated by hsa-miR-122-5p in hepatocytes. Arch Iran Med 20: 165-171, 2017.

26. Khakinezhad Tehrani F, Ranji N, Kouhkan F and Hosseinzadeh S: Apoptosis induction and proliferation inhibition by silibinin encapsulated in nanoparticles in MIA PaCa-2 cancer cells and deregulation of some miRNAs. Iran J Basic Med Sci 23: 469-482, 2020.

27. Zadeh MM, Motamed N, Ranji N, Majidi M and Falahi F: Silibinin-induced apoptosis and downregulation of microRNA-21 and microRNA-155 in MCF-7 human breast cancer cells. J Breast Cancer 19: 45-52, 2016.

This work is licensed under a Creative Commons Attribution-NonCommercial-NoDerivatives 4.0 International (CC BY-NC-ND 4.0) License. 